\title{
Angiogenesis and bone regeneration by mesenchymal stem cell transplantation with danshen in a rabbit model of avascular necrotic femoral head
}

\author{
YUNGANG WU, CHUNWU ZHANG, JIANJING WU, YAN HAN and CHUNLEI WU \\ Department of Orthopedics, The First Affiliated Hospital of Wenzhou Medical University, \\ Wenzhou, Zhejiang 325000, P.R. China
}

Received June 29, 2017; Accepted November 3, 2017

DOI: $10.3892 /$ etm.2019.7556

\begin{abstract}
The present study aimed to explore the potential of combined treatment with mesenchymal stem cells (MSCs) and danshen for angiogenesis and bone regeneration in a rabbit model of avascular necrosis of femoral head (ANFH). A rabbit model of ANFH was established using the Shwartzman reaction with methylprednisolone and Escherichia coli endotoxin injection. Magnetic resonance imaging (MRI) and histopathological examination were used to evaluate the rabbit model of ANFH. The rabbits were randomly divided into the danshen group, the MSCs group, the danshen combined with MSCs group and the model group (treated with physiological saline). The expression level of monocyte chemoattractant protein-1 (MCP-1) and stromal cell-derived factor-1 (SDF-1) were determined by reverse transcription polymerase chain reaction (RT-PCR). The expression level of bone morphogenetic protein-2 (BMP-2) and vascular endothelial growth factor (VEGF) were detected by immunofluorescence and the mRNA expression of BMP-2 and VEGF were detected by RT-PCR. Typical osteonecrosis occurred in the rabbit model of ANFH, which indicated that the model was successfully established. MCP-1 and SDF-1 were significantly increased in the model group compared with the normal group $(\mathrm{P}<0.05)$. Following the administration of MSCs and Salvia miltiorrhiza (danshen), MSCs labeled with 5-bromo-2-deoxyuridine were observed to be gathered in the necrotic area. The increased migration of MSCs to the necrotic area may be due to the upregulated expression of the chemokines MCP-1 and SDF-1. ANFH treated with danshen combined with MSCs may promote
\end{abstract}

Correspondence to: Dr Chunlei Wu, Department of Orthopedics, The First Affiliated Hospital of Wenzhou Medical University, 2 Fuxuexiang Road, Wenzhou, Zhejiang 325000, P.R. China E-mail: wuchunlei0123@126.com

Key words: avascular necrosis of femoral head, mesenchymal stem cells, danshen, monocyte chemoattractant protein-1, stromal cell-derived factor-1, vascular endothelial growth factor, bone morphogenetic protein-2 revascularization by increasing the expression of VEGF and BMP-2 in the femoral head, promoting re-ossification and revascularization. Danshen combined with the transplantation of MSCs may be regarded as a novel therapy for the treatment of ANFH in a clinical setting.

\section{Introduction}

Avascular necrosis of the femoral head (ANFH) is one of the most diagnosed osteoarthritic diseases worldwide (1), it is a chronic disease that typically leads to destruction of the hip joint in patients aged 30-60. It has been estimated that there are 10,000-20,000 new cases of ANFH each year in the USA (2-5). ANFH may be induced by a number of risk factors, including vascular compression, hypertension, thrombosis, alcoholism, chemotherapy and excessive glucocorticoid treatment; however, the pathophysiology of ANFH remains unclear (6-8). Without effective and prompt treatment, progression of the disease may worsen joint dysfunction and even lead to permanent disability (6). The majority of patients exhibit dysfunction of the hip joints within 1-4 years of disease development (9). Once the articular surface of the femur collapses, treatment is extremely challenging and usually ineffective.

Mesenchymal stem cells (MSCs) are adult stem cells that are capable of self-renewal and divergence into multiple lineages, including bone, cartilage, adipose tissue, muscle, tendon and stroma (10). The ability of MSCs to overcome cellular senescence demonstrates a resolution to prolong life span and supports the study of MSC biogenesis on ANFH. MSCs have been isolated from almost every tissue or organ of the human body, including fat, bone marrow, blood, umbilical cord, placenta, lung, skin and skeletal muscle (11). MSC based treatments are regarded as novel methods of ANFH therapy due to the differentiation potential of MSCs and the ease with which they are prepared (12).

Salvia miltiorrhiza (danshen) is a widely used Chinese herbal medicine (13). Danshen may hold promise as a sensitizing agent for chemotherapy and radiotherapy to enhance the cytotoxic effects of anti-cancer agents (14). A number of previous studies have reported that danshen performs a variety of biological activities, including antithrombous, antiplatelet aggregation, moluscicide, antioxidant, antiviral and antitumor 
actions (14-17). However, the underlying mechanisms behind how the active components function still require clarification (15). The present study was performed to investigate the potential therapeutic effect of danshen in ANFH.

The aim of the present study was to establish a rabbit model of methylprednisolone (MP)-induced ANFH using the Shwartzman response and to explore the mechanism of MSCs migration to the necrotic area of the femoral head. The local Shwartzman response has traditionally been produced in rabbits using an intradermal injection of endotoxin followed by an intravenous injection of an agent which activates neutrophils $24 \mathrm{~h}$ later (16). Initially, pathological changes in the femoral head region were detected using imaging and histopathology techniques, including magnetic resonance imaging (MRI) and hematoxylin and eosin (H\&E), Sudan III and phosphotungstic acid hematoxylin (PATH) staining, then the number of MSCs in the femoral head necrotic area was detected. The mRNA expression of monocyte chemoattractant protein-1 (MCP-1) and stromal cell-derived factor-1 (SDF-1) was measured using reverse transcription polymerase chain reaction (RT-PCR). The expression of bone morphogenetic protein-2 (BMP-2) and vascular endothelial growth factor (VEGF) was also measured to determine the ability of danshen in combination with MSCs to promote bone re-ossification and revascularization for the treatment of ANFH. These results were analyzed and the feasibility of clinical treatment using danshen combined with MSCs transplantation for ANFH was evaluated.

\section{Materials and methods}

Experimental animals. A total of 72 male New Zealand rabbits (age, 24 weeks; weight, $2.5 \pm 0.2 \mathrm{~kg}$ ) were purchased from the Experimental Animal Center of Zhejiang University of Traditional Chinese Medicine (Hangzhou, China). The animals were bred and maintained under a $12 \mathrm{~h}$ light/dark cycle with ad libiutm access to food and water. Room temperature and relative humidity were set at $25 \pm 3^{\circ} \mathrm{C}$ and $60 \pm 15 \%$, respectively. All procedures and animal care were approved by the Institutional Animal Care (Hangzhou, China) and Use Ethical Review Committee of Wenzhou Medical University (Wenzhou, China).

Isolation, culture, identification and labeling of MSCs. MSCs from rabbits were isolated and cultured as previously described (17). Briefly, bone marrow was extracted from the rabbit femur and purified by density gradient centrifugation $\left(1,600 \mathrm{x} \mathrm{g} ; 4^{\circ} \mathrm{C} ; 25 \mathrm{~min}\right)$ using Percoll solution (Sangon Biotech Co., Ltd., Shanghai, China). The cells were cultured in Dulbecco's Modified Eagle medium (Gibco; Thermo Fisher Scientific, Inc., Waltham, MA, USA) with $10 \%$ fetal bovine serum (Gibco) and incubated at $37^{\circ} \mathrm{C}$ in humidified air with $5 \% \mathrm{CO}_{2}$ for $24 \mathrm{~h}$. Non-adherent cells were discarded when the medium was refreshed, which was performed every 3-4 days. Following culturing for three passages in vitro, the cells were detached and incubated with the anti-rabbit antibodies, CD34 (1:300; cat. no. bs-2042R) and CD44 (1:300; cat. no. bs-0521R) at $4{ }^{\circ} \mathrm{C}$ overnight. All antibodies were purchased from BIOSS (Beijing, China). Cells were then observed under an inverted fluorescence microscope following immunofluorescence staining at a magnification of x200. Following 3-4 passages, the MSCs were harvested and used for cell implantation. Cultured MSCs were labeled with 5-bromo-2-deoxyuridine (BrdU; 10 mg/l; Sigma-Aldrich; Merck KGaA, Darmstadt, Germany) overnight at $37^{\circ} \mathrm{C}$ according to the manufacturer's protocol prior to transplantation. Following labeling, the cells were washed five times with PBS to remove all excess unbound BrdU. The MSCs were subsequently harvested ( $1 \times 10^{7}$ cells per implantation) and resuspended in IMDM media for further use.

Establishment of a rabbit model of ANFH. A rabbit model of ANFH was established according to the Shwartzman response method with modifications: $20 \mu \mathrm{g} / \mathrm{kg}$ Escherichia coli endotoxin (LPS) was utilized instead of the $50 \mu \mathrm{g} / \mathrm{kg}$ dose utilized in the literature (18). Healthy New Zealand rabbits were separated randomly into the normal $(n=3)$ and model groups ( $\mathrm{n}=7 ; 4$ of 7 rabbits succumbed). A total of $20 \mu \mathrm{g} / \mathrm{kg}$ LPS (Sigma-Aldrich) was injected through an ear vein to induce bone necrosis and this process was repeated after $24 \mathrm{~h}$. Following the second administration of LPS, $20 \mathrm{mg} / \mathrm{kg} \mathrm{MP}$ (Pharmacia and Upjohn; Pfizer Inc, NY, USA) was injected through the gluteus muscle three times with an interval of $24 \mathrm{~h}$ between each dose.

MRI. All rabbits were examined using a GE Signa EXCITE 1.5T MRI machine (GE Healthcare, Chicago, IL, USA). At 3 weeks an orthogonal head coil was placed on the rabbits while they were anesthetized, with its center located on the femoral head. The layer thickness was $3 \mathrm{~mm}$ and the field of view was $240 \mathrm{~mm}^{2}$. The changes in femoral head signal and the surrounding soft tissue were observed.

Histopathological assay. The rabbits were anesthetized using intravenous administration with $3 \%$ sodium pentobarbital (30 $\mathrm{mg} / \mathrm{kg}$ ) in an ear vein and the animals were subsequently sacrificed via air embolism. The left femoral head was divided into two parts along the coronal plane of the central hole and fixed in 10\% formaldehyde solution (Sangon Biotech Co., Ltd., Shanghai, China) at $4^{\circ} \mathrm{C}$ for $48 \mathrm{~h}$, decalcified for 3 months with $0.27 \mathrm{~mol} / \mathrm{l} \mathrm{EDTA}-2 \mathrm{Na}$ (Sangon Biotech Co., Ltd.) at $25^{\circ} \mathrm{C}$ with $\mathrm{pH}$ 7.4. H\&E and Sudan III (performed on $5 \mu \mathrm{m}$ and $20 \mu \mathrm{m}$ sections obtained from the same part of the femoral head, respectively, at room temperature for $15 \mathrm{~min}$ ), and PATH staining (performed on $7 \mu \mathrm{m}$ sections at room temperature for $24 \mathrm{~h}$; all Sigma-Aldrich; Merck KGaA) was performed to observe histopathological changes using a light microscope (Olympus Corporation, Tokyo, Japan). Following H\&E staining, the number of empty bone cells were counted at a magnification, $x 400$ to calculate the empty bone trap rate. Following Sudan III staining, the fat embolism number (number of blood vessels with fat embolization in the femoral head cartilage) was counted by eye using a microscope at magnification, $x 200$. Following PATH staining, the thrombosis number was observed and the thrombosis rate was calculated (thrombosis number/total blood vessels).

$R T$-PCR. The rabbits in the model group were sacrificed at 1 , 2 and 3 weeks following the final injection of MP. RNA was extracted from the right femoral head using a Qiagen RNeasy Mini kit, which included the RNA buffer (cat. no. 74104; Qiagen, Inc., Valencia, CA, USA) after the bone was milled 
Table I. Primer sequences for reverse transcription-quantitative polymerase chain reaction.

\begin{tabular}{lcl}
\hline Gene & Direction & \multicolumn{1}{c}{ Sequence (5'-3') } \\
\hline MCP-1 & F & CCGCCAGGTGGGCTAATA \\
& R & AGCAAGCAGAGCGAGGGT \\
SDF-1 & F & CCCACCATCTACTCCATCA \\
& R & GAAATCGGGAATAGTCAGC \\
VEGF & F & ATGGCAGAAGAAGGAGACA \\
& R & GCCCTGGTGAGGTTTGAT \\
BMP-2 & F & GGAAGAACTGCCAGAAAC \\
& R & GACCTGCTAATCCTCACG \\
$\beta$-actin & F & TCCTGCGTCTGGACCTGG \\
& R & GCCCGACTCGTCATACTCC
\end{tabular}

F, forward; R, reverse; MCP-1, monocyte chemoattractant protein-1; SDF-1, stromal cell-derived factor-1; VEGF, vascular endothelial growth factor; BMP-2, bone morphogenetic protein-2.

(SPEX 6870 Freezer Mill; SPEX SamplePrep, Metuchen, NJ, USA) in liquid nitrogen. Samples were obtained from the femoral heads of the normal group and animals were sacrificed at the same time points as the model group. The sequences of rabbit MCP-1, SDF-1, BMP, VEGF and $\beta$-actin genes were obtained from GenBank (https://www.ncbi.nlm. nih.gov/nuccore). The primer sequences used are listed in Table I. The corresponding primers were synthesized by Sangon Biotech Co., Ltd. (Shanghai, China).

Following RNA extraction from the right femoral head a PrimeScript ${ }^{\mathrm{TM}}$ 1st Strand cDNA Synthesis kit (Takara Bio, Inc., Otsu, Japan) was used to reverse transcribe the RNA into cDNA according to the manufacturers' protocol. RT-PCR was performed according to the following thermocycling conditions: $94^{\circ} \mathrm{C}$ for $3 \mathrm{~min}$, followed by 40 cycles of $94^{\circ} \mathrm{C}$ for $30 \mathrm{sec}$, $52^{\circ} \mathrm{C}$ for $30 \mathrm{sec}$ and $72^{\circ} \mathrm{C}$ for $20 \mathrm{sec}$. The relative quantification of each gene mRNA was calculated relative to the $\beta$-actin mRNA.

Immunohistochemistry assay. The 24 model rabbits were randomly divided into the following four groups ( $\mathrm{n}=6$ in each): Danshen (cat. no. Z33020177-2009; lot no. 0908213; Chiatai Qingchun Bao Pharmaceutical Co. Ltd., Hangzhou China) injection group ( $2 \mathrm{ml}$ per rabbit), the MSCs group (1) $10^{7}$ cells per rabbit), the danshen combined with MSCs group ( $2 \mathrm{ml}$ danshen $+1 \times 10^{7}$ MSCs per rabbit) and the model group, which were administered physiological saline ( $2 \mathrm{ml}$ per rabbit). The BrdU-labeled MSCs were extracted from rabbits through density gradient centrifugation and adherence screening at the third generation as described above. BrdU has been previously confirmed to have no inhibiting effect on MSC proliferation (19). The rabbits were administered their respective treatments in two sides of the femoral artery at 4 weeks following the final injection of MP. All rabbits were administered penicillin 3 days later to prevent infection following treatment. At 3 and 6 weeks following treatment, 3 rabbits from each group were sacrificed. Immunohistochemistry analysis was used to determine the protein expression of VEGF and
BMP-2 and the number of MSCs labeled with BrdU to evaluate the ability of MSCs to migrate to the necrotic area. Femoral head samples were fixed in $10 \%$ formaldehyde for $48 \mathrm{~h}$ at room temperature, cut into 3-4 $\mu \mathrm{m}$ sections and embedded in paraffin (Sangon Biotech Co., Ltd.). For VEGF, BMP-2 and BrdU immunohistochemical staining, femoral head sections were deparaffinized in xylene and dehydrated with graded ethanol. After washing with distilled water, tissue peroxidase was blocked using 3.0\% hydrogen peroxide in methanol for $10 \mathrm{~min}$ at room temperature. For antigen retrieval using citric acid buffer, slides were heated at $120^{\circ} \mathrm{C}$ for $20 \mathrm{~min}$ and then cooled for $15 \mathrm{~min}$ at room temperature. Following washing with PBS solution, slides were incubated with VEGF (1:100; cat no. SC-7269; Santa Cruz Biotechnology, Inc., Dallas, TX, USA), BMP-2 (1:100; cat no. SAB1411278) and BrdU (1:100; cat. no. B2531; both Sigma-Aldrich; Merck KGaA) antibodies at $37^{\circ} \mathrm{C}$ for $1 \mathrm{~h}$. Samples were then washed with PBS in triplicate and incubated with anti-mouse horseradish peroxidase (HRP) conjugated immunoglobulin G (IgG; cat. no. 7076) or anti-rabbit HRP-conjugated IgG antibodies (cat. no. 7074; both CST Biological Reagents Co., Ltd., Shanghai, China) diluted $(1: 2,000)$ by Antibody Diluent Reagent Solution (cat. no. 1956331 A; Thermo Fisher Scientific, Inc.) for $30 \mathrm{~min}$ at $37^{\circ} \mathrm{C}$. Any immune reaction was detected using 3,3'-diaminobenzidine. Sections were then counterstained at room temperature for $10 \mathrm{~min}$ using Meyer's hematoxylin, dehydrated and mounted. The known positive samples were then utilized as positive controls. Following immunohistochemical staining using the Histostain-Plus kit (Mai Bio Co, Ltd., China), the cell number was counted under a light microscope at magnification, $\mathrm{x} 400$.

Statistical analysis. All statistical analyses were performed using SPSS software, version 13 (SPSS, Inc., Chicago, IL, USA). Data are presented as the mean \pm standard deviation and all tests were performed in triplicate. Statistical comparisons between two groups were made using Student's t-test. One-way analysis of variance followed by a post hoc Tukey's test was used to analyze differences among multiple groups. $\mathrm{P}<0.05$ was considered to indicate a statistically significant difference.

\section{Results}

MRI. There was no clear abnormality observed in the normal group femoral heads (Fig. 1A). In T2 weighted imaging, MRI revealed a normal joint space of the femoral head in the rabbit model and an infusion in the joint cavity. The right femoral head exhibited scattered spots in the medium-high signal region and inhomogeneous low signal mixing. MRI images of the model group revealed that the femoral head was not smooth (Fig. 1B).

Histopathology results. H\&E staining revealed that in the normal group, the trabecular bone was complete and arranged, the bone marrow was rich in hematopoietic cells and osteoblasts were observed on the surface of the trabecular bone (Fig. 1C). Cancellous bone near the femoral neck had undergone bone necrosis at week 4 in the model group; this was accompanied by neutrophil aggregation (Fig. 1D). 

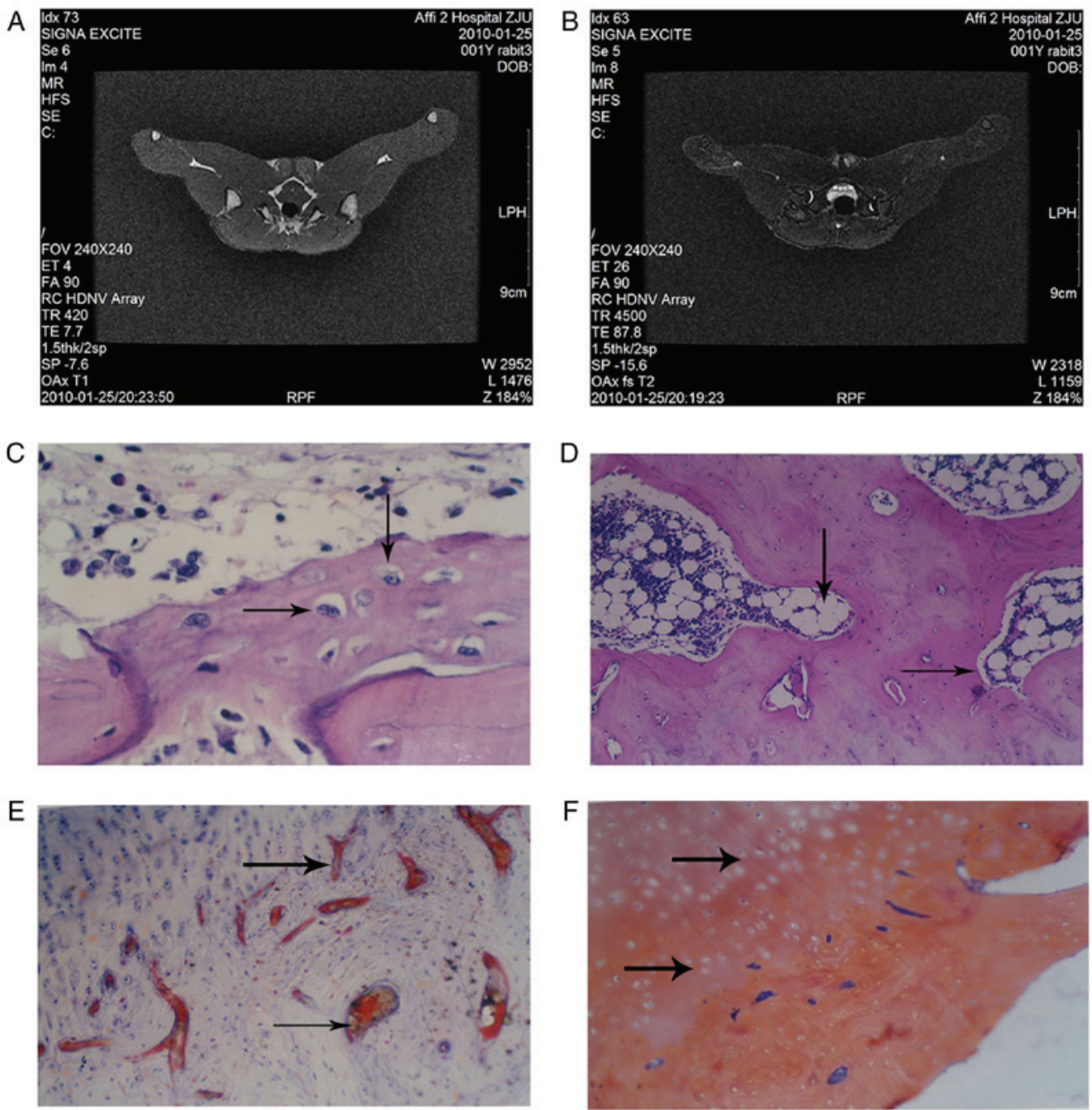

Figure 1. Histopathology of the rabbit tissue in the normal group and the model group. Magnetic resonance imaging scans were performed on rabbits in the (A) normal and (B) model group and representative images are presented. Hematoxylin and eosin staining was performed on tissue from the (C) normal group (arrows indicate osteoblasts) and the (D) model group (arrows indicate empty bone cells). (E) Sudan III staining was performed on tissue from the model group (arrows indicate fat emboli). (F) Phosphotungstic acid hematoxylin staining was performed on tissue from the model group (arrows indicate fibrin emboli). All the images were obtained at a magnification, $\mathrm{x} 100$.

Sudan III staining demonstrated that there was no notable steatosis or lipid embolism in the liver and kidneys in the model group (Fig. 1E). Lipid droplets filled with cellular fluid were observed and the nucleus appeared misshapen under pressure. In the normal group, the lipid droplets in the bone cells were not clear, the fat cells in the bone marrow were smaller compared with the model group and the hematopoietic cells were observed in the voids of the bone marrow (data not shown).

The model group exhibited a large number of fibrin embolus in the femoral head cartilage, which was observed by PATH staining (Fig. 1F). The presence of fibrin embolus was less common in the control group (data not shown).

The empty bone rate, fat embolism rate and thromboembolism rate were calculated according to the aforementioned results and are presented in Fig. 2. The empty bone, fat embolism and thromboembolism rates were all significantly increased in the model group compared with the normal group $(\mathrm{P}<0.05)$.

BrdU-labeled MSCs are increased in the model group compared with the normal group. At 3 weeks, few

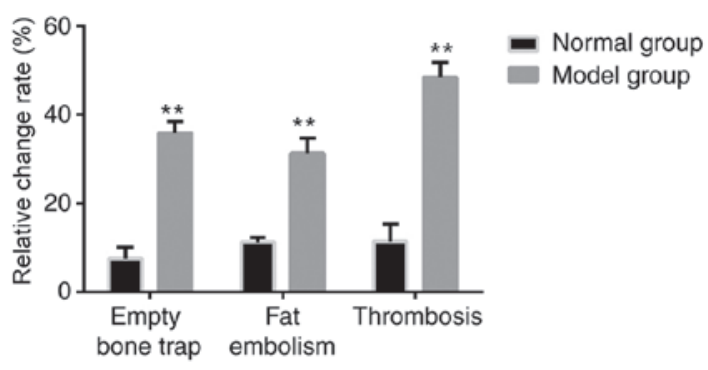

Figure 2. Proportion of empty bone, fat emboli and thromboembolism were compared between the groups. Using the stained tissue, the proportion of empty bone, fat emboli and thromboembolism was determined in the normal and model groups. ${ }^{* *} \mathrm{P}<0.05$ vs. normal group.

BrdU-labeled MSCs were detected in the normal group (Fig. 3A), whereas a greater number of BrdU-labeled MSCs were observed in the model group (Fig. 3B). The nuclei were stained brown. There was a significantly higher number of MSCs observed in the model group compared with the normal group $(\mathrm{P}<0.05$; Fig. $3 \mathrm{C})$. 
A
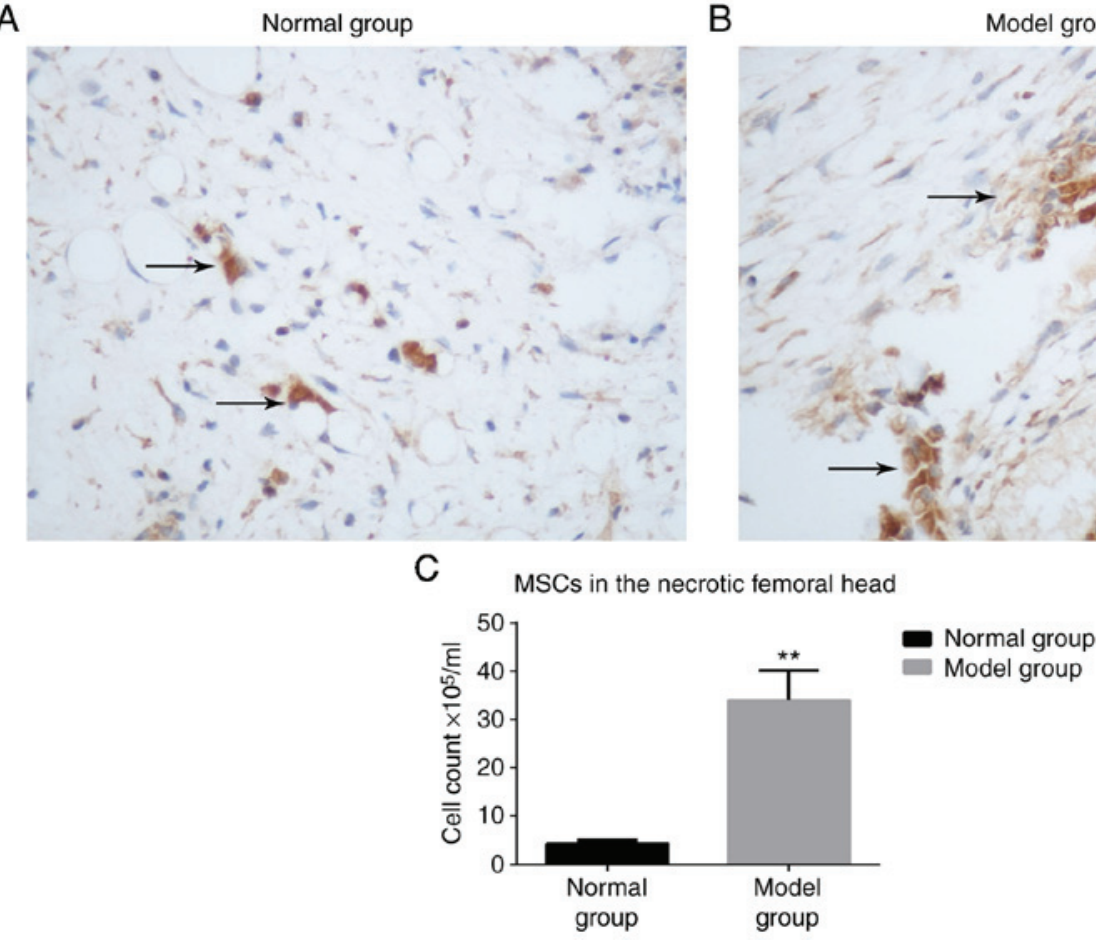

B

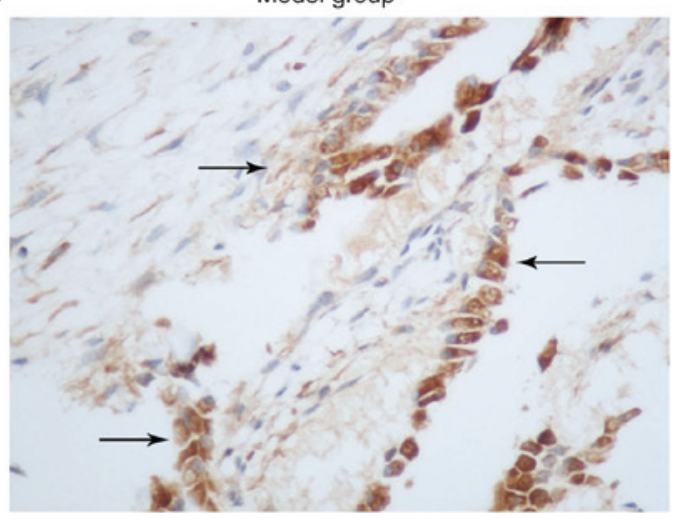

Figure 3. Immunohistochemistry of MSCs. Immunohistochemistry staining of the (A) normal and (B) model groups (arrows indicate 5-bromo-2-deoxyuridine-labeled MSCs in the femoral head; magnification, x200). (C) The number of MSCs present were counted and the results are indicated as a bar graph. ${ }^{* *} \mathrm{P}<0.05$ vs. normal group. MSC, mesenchymal stem cell.

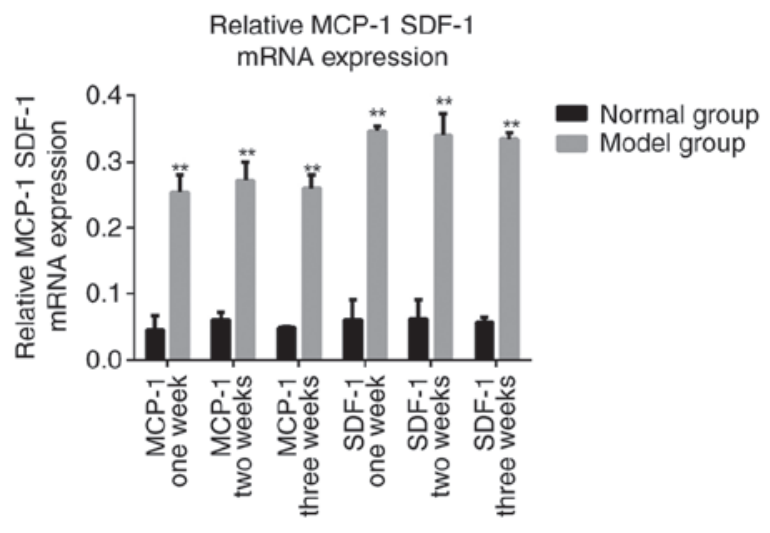

Figure 4. Relative MCP-1 and SDF-1 mRNA expression. The chemokine expression of MCP-1 and SDF-1, normalized to $\beta$-actin was measured at weeks 1,2 and 3 following the final injection of methylprednisolone to develop the rabbit model of avascular necrosis of femoral head. ${ }^{* *} \mathrm{P}<0.05$ vs. the normal group. MCP-1, monocyte chemoattractant protein-1; SDF-1, stromal cell-derived factor-1.

Expression of MCP-1 and SDF-1 are significantly increased in the model group. RT-PCR revealed that the chemokine expression of MCP-1 and SDF-1 was significantly increased in the model group compared with the normal group at all time points measured $(\mathrm{P}<0.05$; Fig. 4). These results suggest that in the necrotic femoral head MCP-1 and SDF-1 mRNA expression was increased and continued for an extended period of time.

Expression of BMP-2 and VEGF. At 3 weeks following the final administration of MSCs and danshen, the mRNA expression of BMP-2 and VEGF in the femoral head and surrounding area was significantly increased in the groups treated with
MSCs, danshen, or a combination of the two compared with the model group $(\mathrm{P}<0.05$; Fig. 5), Similar results were observed at 6 weeks. There was no significant difference observed between the results at 3 weeks and the results at 6 weeks. These results suggest that the expression of BMP-2 and VEGF mRNA is increased by treatment with danshen or MSCs, but this difference is not time-dependent. The group that received MSC in combination with danshen demonstrated a significant increase in the level of BMP-2 compared with the single treatment groups $(\mathrm{P}<0.05)$. The mRNA expression results were consistent with the immunohistochemistry results.

Protein expression of MCP-2 and VEGF in the femoral head. Immunohistochemistry staining was performed on tissue samples from each treatment group at 3 and 6 weeks following treatment (Fig. 6) The immunohistochemistry results revealed that the protein expression rates of BMP-2 and VEGF in the femoral head at 3 and 6 weeks following treatment were significantly higher in all groups treated with MSCs and/or danshen compared with the model group ( $\mathrm{P}<0.05$; Fig. $5 \mathrm{~B})$. However there was no significant difference between the BMP-2 and VEGF expression in all of the groups at week 3 compared with week 6 . This suggests that the expression of BMP-2 and VEGF may be increased by the injection of danshen and MSCs, either individually or in combination, but this is not time-dependent. The positive expression rate of BMP-2 and VEGF in the MSCs and danshen group was significantly increased compared with that of the MSCs or danshen only groups $(\mathrm{P}<0.05)$. At week 6 the MSCs with danshen group had a significantly increased BMP-2 expression compared with week $3(\mathrm{P}<0.05)$, however this was not observed for VEGF expression. This suggests that the effect of danshen combined with MSCs was higher 

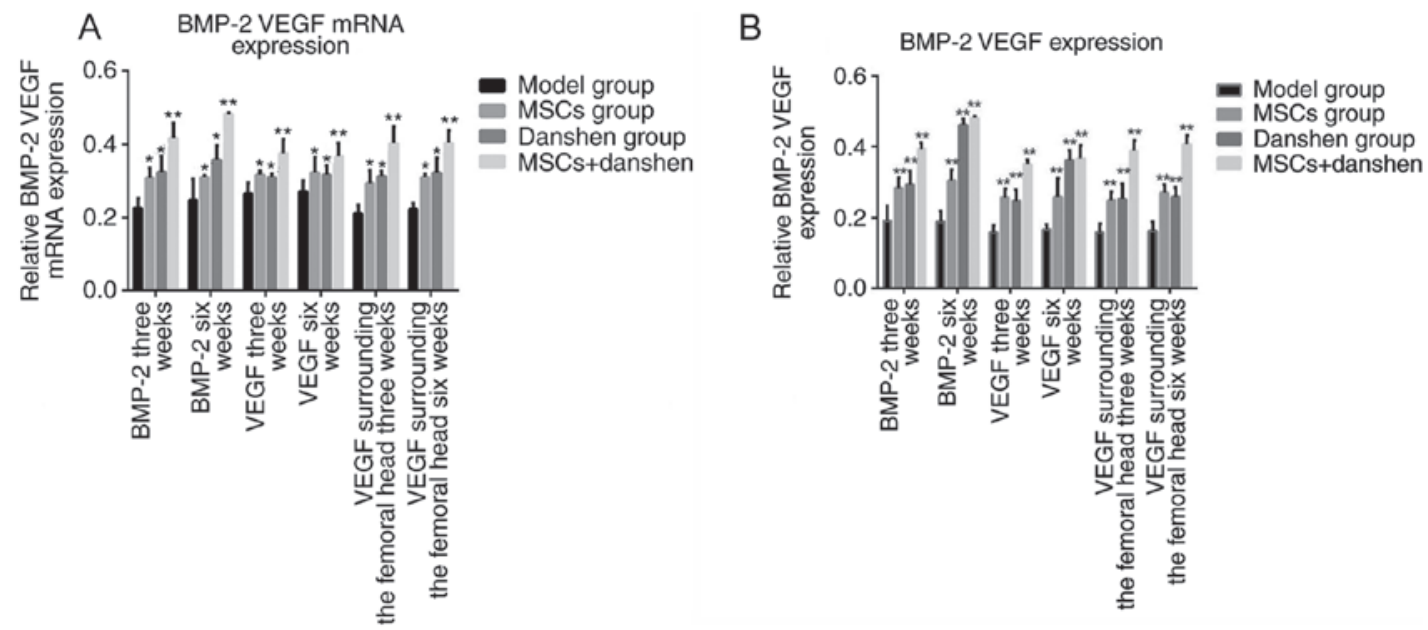

Figure 5. Expression of BMP-2 and VEGF. (A) The mRNA expression of BMP-2 and VEGF was measured by reverse transcription-quantitative polymerase chain reaction at 3 and 6 weeks following treatment with danshen and MSCs alone or in combination. (B) The results of the immunohistochemistry staining were quantified and presented as a bar chart. ${ }^{*} \mathrm{P}<0.05$. vs. the model group; ${ }^{* *} \mathrm{P}<0.01$. vs. the model group. BMP-2, bone morphogenetic protein-2; VEGF, vascular endothelial growth factor; MSC, mesenchymal stem cell.

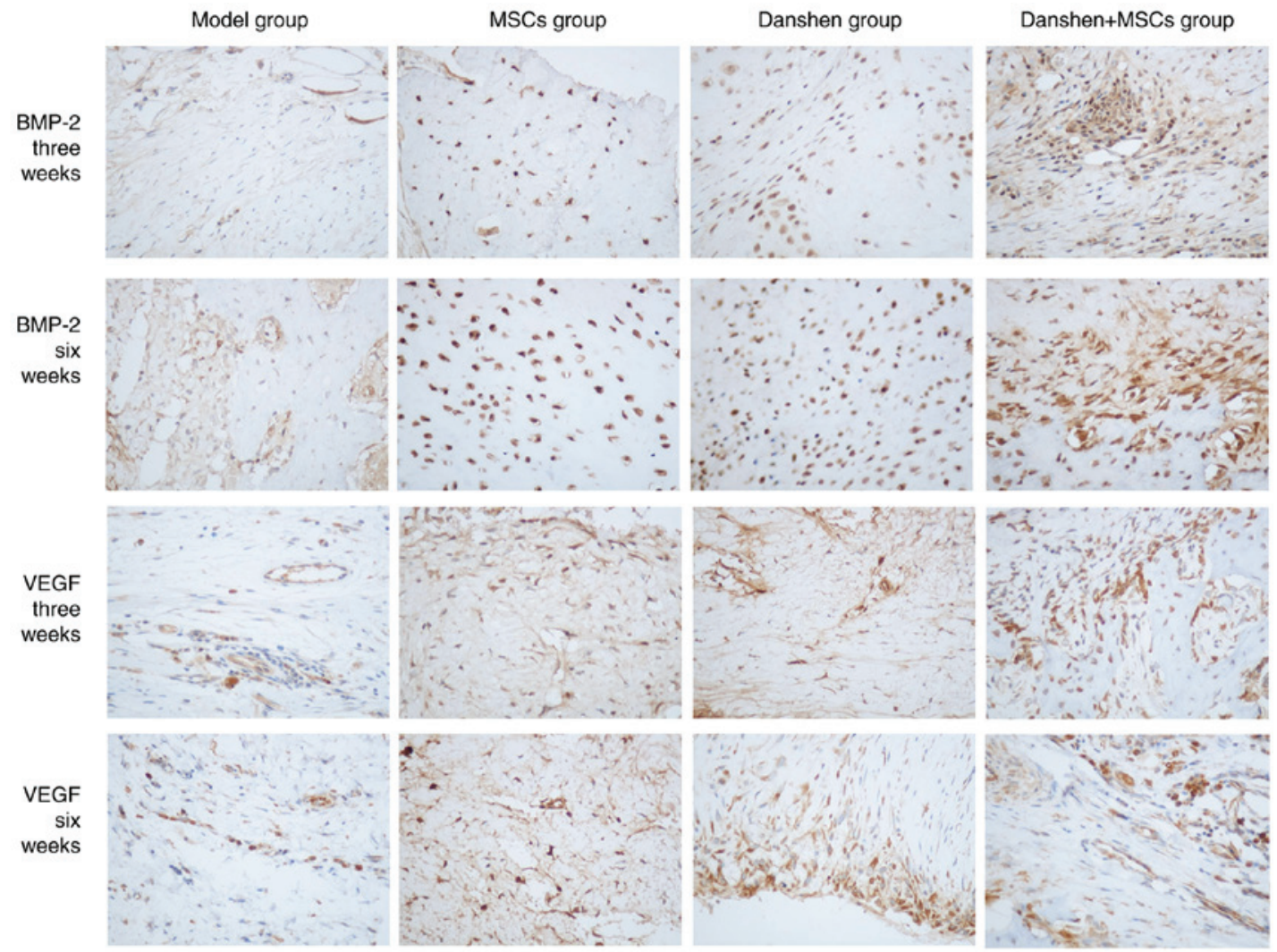

Figure 6. Immunohistochemistry of VEGF and BMP-2. Tissue samples underwent immunohistochemistry staining to detect the expression of VEGF and BMP-2 at different time points following treatment with danshen and MSCs alone or in combination. Representative images are presented (magnification, x100). BMP-2, bone morphogenetic protein-2; VEGF, vascular endothelial growth factor; MSC, mesenchymal stem cell.

compared with a single administration of either danshen or MSCs and that BMP-2 expression increased over an extended period of time.

\section{Discussion}

The rabbit model of hormone-induced ANFH has been used to study ANFH for a number of years. At present, there are many different types of model available to study ANFH including the serological necrosis model and Shwartzman response model (18-21). As ANFH is not reversible it is important to develop novel therapeutic agents for its early diagnosis and treatment. Previously, hormones have been used to induce ANFH with limited success (22). In the present study, the improved Shwartzman response model (18) was used to develop ANFH via administration of MP following LPS, 
which induced experimental femoral head necrosis with a low mortality rate. These aforementioned improved models are of great value in the future study of the ANFH. In clinical practice, MRI is the most accurate imaging method available and is particularly useful to diagnose ANFH at its early stage when changes are only visible in the bone marrow (23). In the present study MRI scans revealed an edema and a spot-like high level signal in the right femoral head. These results are consistent with the image characteristics of ANFH (24). The histopathological methodologies used in the present study, including H\&E, Sudan III and PATH staining revealed that the rabbit model of ANFH was successfully established.

Chemokines are small molecular weight proteins $(8-13 \mathrm{kDa})$ and are categorized into four different families (CC, CXC, $\mathrm{CX} 3 \mathrm{C}$ and $\mathrm{C}$ ) based on the presence of $\mathrm{NH}_{2}$ terminal cysteine motifs (24). Chemokine receptors are typical $\mathrm{G}$ protein-coupled transmembrane proteins (24). When the associated chemokine ligand binds with the chemokine receptor, G $\alpha 1$ and $G \beta-\gamma$ subunits dissociate and activate phosphatidylinositol 3-kinase and small Rho guanosine triphosphatase, which leads to cellular calcium influx (25). Chemokine receptors are typically expressed by leukocyte subsets and immune cells generally carry different sets of chemokine receptors (26). The roles of chemokines relevant to orthopedic implant debris includes pro-inflammatory cytokine production, pyroptosis, apoptosis, angiogenesis and collagen production, which act together to product aseptic bone reabsorption around implants (27). In the present study RT-PCR revealed that the expression of MCP-1 and SDF-1 mRNA was significantly increased in the model group compared with the normal group, which may be associated with the repair of the femoral head as a previous study revealed that MCP-1 mRNA and SDF-1 may attract MSCs to the necrotic area by chemotaxis and then use the MSCs to repair injury (25). However, the specific repair mechanisms require further study to be fully understood.

Osteoblasts, osteoclasts and mesenchymal stem cells secrete BMP-2 and VEGF for bone growth and repair. Previous studies have demonstrated that the combined use of BMP-2 and VEGF is superior to their use alone, as they have a synergistic effect in promoting bone regeneration and vascularization (28-30). In the present study, immunohistochemical detection and RT-PCR revealed that BMP-2 and VEGF had a significantly increased expression in groups administered the combined treatment of danshen and MSCs compared with the single treatment groups. The combined use of danshen and MSCs may have synergistic effects in promoting the attachment, proliferation, osteogenic and angiogenic activities of MSCs. It was observed that treatment with MSCs or danshen separately induced a significant increase in BMP-2 and VEGF expression compared with the model group and the danshen and MSCs group had significantly increased expression of BMP-2 and VEGF compared with the single treatment groups. This indicates that the effect of danshen combined with MSCs on femoral artery intervention was better than danshen or MSCs treatment alone. The role of BMP-2 and VEGF in promoting bone and vessel formation in vivo may be due to its induction of the proliferation and osteogenic and angiogenic differentiation in bone MSCs. High expression of BMP-2 and VEGF may accelerate re-vascularization and re-ossification in patients with ANFH.

Clinicians have suggested that an ideal treatment should consider pain relief, preservation or restoration of the integrity of the femoral head and prevention of deterioration of the hip (31). Several treatment methods and procedures have been performed to try and cure ANFH, including core decompression (32-34), electrical stimulation $(35,36)$, transtrochanteric rotational osteotomy (37), non-vascularized structural grafting $(38,39)$ and a vascularized bone graft $(40,41)$. However, none of these offer a complete cure $(42,43)$ and to the best of our knowledge there is no treatment currently available that effectively reduces painful necrotic bone without damaging native hip geometry. Therefore, the development of a therapeutic treatment that is low risk, minimally invasive, low cost, easily applied and effective is currently of major focus in the orthopedic field. Due to developments in tissue engineering, stem cell transplantation has become a good alternative for tissue repair and is an applicable methodology for ANFH due to the ability of MSCs to differentiate into osteoblasts, chondrocytes, muscle and fat cells (44). To improve the therapeutic effect and reduce trauma, the present study treated ANFH with the co-transplantation of MSCs and danshen injection. The aim of gene therapy for osteonecrosis disease is bone and vessel regeneration and previous studies have confirmed that bone and vessel regeneration is mediated by BMP-2 and VEGF, which are critical for bone repair $(28,45)$. Previous studies have reported that BMP-2 and VEGF may promote self-repair of necrotic femoral heads $(46,47)$, in the present study, it was observed that MSCs combined with Danshen promoted BMP-2 and VEGF expression, which is regarded as a critical factor to promote revascularization and re-ossification.

In the present study it was confirmed that LPS and MP induce ANFH in a rabbit model. Additionally, following the administration of MSCs and danshen into the femoral artery, a significantly increased number of BrdU-labeled MSCs were observed and there was a significant increase in the expression of MCP-1 and SDF-1, BMP-2 and VEGF compared with the groups without treatment. When transferred to a clinical setting, these results suggest that MSCs combined with danshen may be used as a novel approach to treat ANFH, as they increase the expression of BMP-2 and VEGF in the necrotic bone area and therefore accelerate the process of revascularization and re-ossification.

\section{Acknowledgements}

Not applicable.

\section{Funding}

The present study was supported by Wenzhou Medical University (grant no. 2008YB011).

\section{Availability of data and materials}

The datasets used and/or analyzed during the current study are available from the corresponding author on reasonable request.

\section{Authors' contributions}

YW and CW designed the present study, and CZ, JW and $\mathrm{YH}$ were responsible for data access and analysis. All authors collaborated to interpret results and develop the manuscript. 


\section{Ethics approval and consent to participate}

All procedures and animal care were approved by the Institutional Animal Care and Use Ethical Review Committee of Wenzhou Medical University.

\section{Patient consent for publication}

Not applicable.

\section{Competing interests}

The authors declare that they have no competing interests.

\section{References}

1. Zhao D, Cui D, Wang B, Tian F, Guo L, Yang L, Liu B and Yu, X Treatment of early stage osteonecrosis of the femoral head with autologous implantation of bone marrow-derived and cultured mesenchymal stem cells. Bone 50: 325-330, 2012.

2. Ohzono K, Saito M, Takaoka K, Ono K, Saito S, Nishina T and Kadowaki T: Natural history of nontraumatic avascular necrosis of the femoral head. J Bone Joint Surg Br 73: 68-72, 1991.

3. Arlet J: Nontraumatic avascular necrosis of the femoral head. Past, present, and future. Clin Orthop Relat Res 277: 12-21, 1992.

4. Mankin HJ: Nontraumatic necrosis of bone (osteonecrosis). N Engl J Med 326: 1473-1479, 1992.

5. Roshan A and Ram S: The neglected femoral neck fracture in young adults: Review of a challenging problem. Clin Med Res 6 : 33-39, 2008

6. Ciapetti G, Granchi D, Fotia C, Savarino L, Dallari D, Del Piccolo N, Donati DM and Baldini N: Effects of hypoxia on osteogenic differentiation of mesenchymal stromal cells used as a cell therapy for avascular necrosis of the femoral head. Cytotherapy 18: 1087-1099, 2016.

7. Tabatabaee RM, Saberi S, Parvizi J, Mortazavi SM and Farzan M: Combining Concentrated autologous bone marrow stem cells injection with core decompression improves outcome for patients with early-stage osteonecrosis of the femoral head: A comparative study. J Arthroplasty 30 (Suppl 9): S11-S15, 2015.

8. Song HM, Wei YC, Li N, Wu B, Xie N, Zhang KM, Wang SZ and Wang HM: Effects of Wenyangbushen formula on the expression of VEGF, OPG, RANK and RANKL in rabbits with steroid-induced femoral head avascular necrosis. Mol Med Rep 12: 8155-8161, 2015.

9. Rajpura A, Wright AC and Board TN: Medical management of osteonecrosis of the hip: A review. Hip Int 21: 385-392, 2011.

10. Zhai L, Sun N, Zhang B, Liu ST, Zhao Z, Jin HC, Ma XL and Xing GY: Effects of focused extracorporeal shock waves on bone marrow mesenchymal stem cells in patients with avascular necrosis of the femoral head. Ultrasound Med Biol 42: 753-762, 2016.

11. Kim N and Cho SG: Clinical applications of mesenchymal stem cells. Korean J intern Med 28: 387-402, 2013.

12. Wen Q, Zhou C, Luo W, Zhou M and Ma L: Pro-osteogenic effects of fibrin glue in treatment of avascular necrosis of the femoral head in vivo by hepatocyte growth factor-transgenic mesenchymal stem cells. J Transl Med 12: 114, 2014.

13. Ye YT, Zhong W, Sun P, Wang D, Wang C, Hu LM and Qian JQ: Apoptosis induced by the methanol extract of salvia miltiorrhiza bunge in non-small cell lung cancer through PTEN-mediated inhibition of PI3K/Akt pathway. J Ethnopharmacol 200: 107-116, 2017.

14. Park CH, Shin SH, Lee EK, Kim DH, Kim MJ, Roh SS, Yokozawa T and Chung HY: Magnesium lithospermate B from salvia miltiorrhiza bunge ameliorates aging-induced renal inflammation and senescence via NADPH oxidase-mediated reactive oxygen generation. Phytother Res 31: 721-728, 2017.

15. Zhou L, Zuo Z and Chow MS: Danshen: An overview of its chemistry, pharmacology, pharmacokinetics, and clinical use. J Clin Pharmacol 45: 1345-1359, 2005.

16. Argenbright LW and Barton RW: The Shwartzman response: A model of ICAM-1 dependent vasculitis. Agents Actions 34: 208-210, 1991.
17. Xia CS, Zuo AJ, Wang CY and Wang YZ: Isolation of rabbit bone marrow mesenchymal stem cells using density gradient centrifugation and adherence screening methods. Minerva Medica 104: 519-525, 2013

18. Yamamoto T, Hirano K, Tsutsui H, Sugioka Y and Sueishi K: Corticosteroid enhances the experimental induction of osteonecrosis on rabbits with Shwartzman reaction. Clin Orthop Relat Res 316: 235-243, 1995.

19. Saeed H, Abdallah BM, Ditzel N, Catala-Lehnen P, Qiu W, Amling $M$ and Kassem M: Telomerase-deficiency-related bone loss is caused by intrinsic impairment of mesenchymal stem cell (MSC) functions and increased osteoclastogenesis due to pro-inflammatory micro-environment. Bone 47: S39, 2010.

20. Wang D, Wang G, Liu M, Sun L, Zong W, Jiang H, Zhang H, Li H, Gong J and Sun S: A novel animal model of osteonecrosis of the femoral head induced using a magnetic resonance imaging-guided argon-helium cryotherapy system. Exp Ther Med 7: 1525-1528, 2014.

21. Wen Q, Ma L, Chen YP, Yang L, Luo W and Wang XN: A rabbit model of hormone-induced early avascular necrosis of the femoral head. Biomed Environ Sci 21: 398-403, 2008.

22. Nowak DA and Yeung J: Steroid-induced osteonecrosis in dermatology: A review. J Cutan Med Surg 19: 358-360, 2015.

23. Li Z, Liao W, Zhao Q, Liu M, Xia W, Yang Y and Shao N.: Angiogenesis and bone regeneration by allogeneic mesenchymal stem cell intravenous transplantation in rabbit model of avascular necrotic femoral head. J Surg Res 183: 193-203, 2013.

24. Bartonicek J, Vavra J and Bartoska R: Operative treatment of avascular necrosis of the femoral head after slipped capital femoral epiphysis. Arch Orthop Trauma Surg 131: 497-502, 2011.

25. Zlotnik A and Yoshie O: Chemokines: A new classification system and their role in immunity. Immunity 12: 121-127, 2000.

26. Fritz EA, Glant TT, Vermes C, Jacobs JJ and Roebuck KA: Chemokine gene activation in human bone marrow-derived osteoblasts following exposure to particulate wear debris. J Biomed Mater Res A 77: 192-201, 2006.

27. Marra F and Tacke F: Roles for chemokines in liver disease. Gastroenterology 147: 577-594 e571, 2014.

28. Wasmuth HE, Tacke F and Trautwein C: Chemokines in liver inflammation and fibrosis. Semin Liver Dis 30: 215-225, 2010.

29. Zhang C, Wang KZ, Qiang H, Tang YL, Li Q, Li M and Dang XQ: Angiopoiesis and bone regeneration via co-expression of the hVEGF and hBMP genes from an adeno-associated viral vector in vitro and in vivo. Acta Pharmacol Sin 31: 821-830, 2010.

30. Young S, Patel ZS, Kretlow JD, Murphy MB, Mountziaris PM, Baggett LS, Ueda H, Tabata Y, Jansen JA, Wong $M$ and Mikos AG: Dose effect of dual delivery of vascular endothelial growth factor and bone morphogenetic protein-2 on bone regeneration in a rat critical-size defect model. Tissue Eng Part A 15: 2347-2362, 2009.

31. Zhang C, Ma J, Li M, Li XH, Dang XQ and Wang KZ: Repair effect of coexpression of the hVEGF and hBMP genes via an adeno-associated virus vector in a rabbit model of early steroid-induced avascular necrosis of the femoral head. Transl Res 166: 269-280, 2015.

32. Aldridge JM III and Urbaniak JR: Avascular necrosis of the femoral head: Role of vascularized bone grafts. Orthop Clin North Am 38: 13-22, v, 2007.

33. Fairbank AC, Bhatia D, Jinnah RH and Hungerford DS: Long-term results of core decompression for ischaemic necrosis of the femoral head. J Bone Joint Surg Br 77: 42-49, 1995.

34. Koo KH, Kim R, Ko GH, Song HR, Jeong ST and Cho SH: Preventing collapse in early osteonecrosis of the femoral head. A randomised clinical trial of core decompression. J Bone Joint Surg Br 77: 870-874, 1995.

35. Mont MA, Carbone JJ and Fairbank AC: Core decompression versus nonoperative management for osteonecrosis of the hip. Clin Orthop Relat Res 324: 169-178, 1996.

36. Aaron RK, Lennox D, Bunce GE and Ebert T: The conservative treatment of osteonecrosis of the femoral head. A comparison of core decompression and pulsing electromagnetic fields. Clin Orthop Relat Res 249: 209-218, 1989.

37. Bassett CA, Schink-Ascani M and Lewis SM: Effects of pulsed electromagnetic fields on Steinberg ratings of femoral head osteonecrosis. Clin Orthop Relat Res 246: 172-185, 1989.

38. Sugioka Y, Hotokebuchi T and Tsutsui H: Transtrochanteric anterior rotational osteotomy for idiopathic and steroid-induced necrosis of the femoral head. Indications and long-term results. Clin Orthop Relat Res 277: 111-120, 1992. 
39. Boettcher WG, Bonfiglio M and Smith K: Non-traumatic necrosis of the femoral head. II. Experiences in treatment. J Bone Joint Surg Am 52: 322-329, 1970.

40. Buckley PD, Gearen PF and Petty RW: Structural bone-grafting for early atraumatic avascular necrosis of the femoral head. J Bone Joint Surg Am 73: 1357-1364, 1991.

41. Kirschenbaum IH, Vernace JV, Booth RE Jr, Balderston RA and Rothman RH: Total hip arthroplasty for osteonecrosis. Semin Arthroplasty 2: 234-240, 1991

42. Katz RL, Bourne RB, Rorabeck CH and McGee H: Total hip arthroplasty in patients with avascular necrosis of the hip. Follow-up observations on cementless and cemented operations. Clin Orthop Relat Res 281: 145-151, 1992.

43. Plakseychuk AY, Kim SY, Park BC, Varitimidis SE, Rubash HE and Sotereanos DG: Vascularized compared with nonvascularized fibular grafting for the treatment of osteonecrosis of the femoral head. J Bone Joint Surg Am 85-A: 589-596, 2003.

44. González Della Valle A, Bates J, Di Carlo E and Salvati EA: Failure of free vascularized fibular graft for osteonecrosis of the femoral head: A histopathologic study of 6 cases. J Arthroplasty 20: 331-336, 2005.
45. Duran JM, Makarewich CA, Sharp TE, Starosta T, Zhu F, Hoffman NE, Chiba Y, Madesh M, Berretta RM, Kubo H and Houser SR: Bone-derived stem cells repair the heart after myocardial infarction through transdifferentiation and paracrine signaling mechanisms. Circ Res 113: 539-552, 2013.

46. Zhang HX, Zhang XP, Xiao GY, Hou Y, Cheng L, Si M, Wang SS, $\mathrm{Li}$ YH and Nie L: In vitro and in vivo evaluation of calcium phosphate composite scaffolds containing BMP-VEGF loaded PLGA microspheres for the treatment of avascular necrosis of the femoral head. Mater Sci Eng C Mater Biol Appl 60: 298-307, 2016.

47. Street J, Bao M, deGuzman L, Bunting S, Peale FV Jr, Ferrara N, Steinmetz H, Hoeffel J, Cleland JL, Daugherty A, et al: Vascular endothelial growth factor stimulates bone repair by promoting angiogene-sis and bone turnover. Proc Natl Acad Sci USA 99: 9656-9661, 2002.

cc) (i) (9) This work is licensed under a Creative Commons

EY NO NO Attribution-NonCommercial-NoDerivatives 4.0 International (CC BY-NC-ND 4.0) License. 